\title{
Trends in Salmonella enteric serovar Typhi in Nairobi, Kenya from 2004 to 2006
}

\author{
Doris M. Mengo ${ }^{1}$, Sam Kariuki ${ }^{2,}$ Ann W. T. Muigai ${ }^{3}$ Gunturu N. Revathi ${ }^{4}$ \\ ${ }^{1}$ Institute of Tropical Medicine and Infectious Diseases, Jomo Kenyatta University of Agriculture and Technology, PO Box \\ 62000-00200, Nairobi, Kenya \\ ${ }^{2}$ Center for Microbiology Research, Kenya Medical Research Institute, PO Box 5484000200 Nairobi, Kenya \\ ${ }^{3}$ Jomo Kenyatta University of Agriculture and Technology, PO Box 62000-00200 Nairobi, Kenya \\ ${ }^{4}$ Division of Microbiology, Department of Pathology, The Aga Khan University Hospital, Nairobi, Kenya
}

\begin{abstract}
Background: Typhoid fever is a global health problem. The World Health Organization (WHO) estimates that the current annual global burden of typhoid is approximately 22 million new cases, $5 \%$ of which are fatal.

Methodology: To assess the trends in antibiotic resistance in 100 Salmonella enterica serovar Typhi strains were isolated from the blood of patients in Nairobi, Kenya, from 2004 to 2006. All isolates were tested against ampicilin, chloramphenic, nalidixic acid, ciprofloxacin, cotrimoxazole, cefuroxime, cefriaxone, amoxycillin/clavulanic acid, tetracycline and gentamycin. Susceptibility and resistance were determined using MIC and disk diffusion tests.

Results: From 2004 to 2006 a total of 100 strains were studied; 70\% of the isolates were multidrug resistant (MDR) while 15\% of the isolates were sensitive to all drugs tested. Of 13 isolates that were resistant to ciprofloxacin and nalidixic acid by disk diffusion, 11 had an MIC of 0 . $25 \mu \mathrm{g} / \mathrm{ml}$ while two isolates had an MIC of $1.00 \mu \mathrm{g}, / \mathrm{ml}$. Resistance in ampicillin decreased from $88 \%$ in 2004 to $64 \%$ in 2005 ; this increased to $76 \%$ in 2006 . Similar trends were observed for four other antibiotics tested.

Conclusion: The prescription of first-line antibiotics used in the treatment of $S$. Typhi should be stopped temporarily. Drugs such as cipfloxacin would be useful in the treatment of typhoid caused by MDR $S$. Typhi. There is need to monitor the resistance in flouroquinolones as resistance to these drugs has been observed and they are the current drugs used to treat typhoid.
\end{abstract}

Key words: quinolone; multidrug resistance; disk diffusion; minimum inhibitory concentration (MIC)

J Infect Dev Ctries 2010; 4(6):393-396.

(Received 14 September 2009 - Accepted 16 March 2010)

Copyright $\odot 2010$ Mengo et al. This is an open-access article distributed under the Creative Commons Attribution License, which permits unrestricted use, distribution, and reproduction in any medium, provided the original work is properly cited.

\section{Introduction}

Typhoid fever is a global health problem [1]. The World Health Organization (WHO) estimates that the current annual global burden of typhoid is approximately 22 million new cases, $5 \%$ of which are fatal [2,3]. Typhoid fever is endemic in most parts of Central America, and large epidemics occur in many parts of Southeast Asia, including India, Pakistan, Bangladesh, Vietnam, Malaysia, Indonesia, China and Tajikistan [4]. Clinically apparent bacteraemic $S$. Typhi infection in children aged less than three years has been described in Bangladesh, India, Jordan, and Nigeria [5].

Since 1997 increasing numbers of multidrugresistant (MDR) $S$. Typhi have been isolated from patients in outbreaks in Nairobi and Embu, Kenya, which were related to damaged water supply systems [6]. From these outbreaks MDR $S$. Typhi were sensitive to nalidixic acid and ciprofloxacin but the minimum inhibition concentrations (MICs) were ten times higher than for the non-MDR strains.

The antibiotics that form the mainstay of therapy for typhioid patients in developing countries are ampicilin, co-trimoxazole and chloramphenicol. Due to the increasing resistance to antibacterials used traditionally for therapy, the use of fluoroquinolones, such as ciprofloxacin and ofloxacin, for the treatment of typhoid has become more common in the Asian countries. This in turn has led to an increase in fluoroquinolone resistance in $S$. Typhi [9].

Sporadic cases of ciprofloxacin treatment failure in typhoid fever have been reported in Europe and more recently, in Asia [10,11]. Njinkeng et al. (2005) report that MDR $S$. Typhi and nalidixic acid resistant $S$. Typhi (NARST) strains were found in Cameroon, Central Africa. NARST have also been reported in East Africa [8]. The aim of this study was to characterize $S$. Typhi using antibiotic susceptibility 
patterns. The $S$. Typhi studied was isolated from patients presenting to the Aga Khan University and the Kenyatta National Hospitals in Nairobi Kenya, suspected to have typhoid fever.

\section{Materials and methods}

Study Design

A descriptive, cross-sectional study design was used.

Setting

Isolates of $S$. Typhi were obtained from stock cultures at the Center for Microbiology Research at KEMRI, Nairobi. The isolates had originated from blood cultures of patients with suspected cases of typhoid fever who had presented themselves to the Aga Khan University Hospital and the Kenyatta National Hospital in Nairobi, Kenya.

\section{Bacterial strains}

One hundred $S$. Typhi isolates were obtained from blood cultures of patients with suspected cases of typhoid fever who had presented themselves to the Aga Khan University Hospital and the Kenyatta National Hospital in Nairobi, Kenya. Bacterial strains had been isolated between 2004 and 2006.

\section{Antibiotic susceptibility testing}

Antimicrobial susceptibility testing was performed using a disc diffusion method according to CLSI guidelines (CLSI, 2005). Escherichia coli ATCC $25922^{\circledR}$ (with known disc zones and MICs) was used as a control for growth and potency of antibiotics. The antibiotic discs, (all from Himedia, Maharashtra, India) contained ampicillin $(30 \mu \mathrm{g})$, tetracycline $(30 \mu \mathrm{g})$, cotrimoxazole $(1: 25 \mu \mathrm{g})$, chloramphenicol $(30 \mu \mathrm{g})$, gentamicin $(10 \mu \mathrm{g})$, amoxycillin/clavulanic acid $(30 \mu \mathrm{g})$, ciprofloxacin (5 $\mu \mathrm{g})$, cefuroxime $(30 \mu \mathrm{g})$, ceftriaxone $(30 \mu \mathrm{g})$, and nalidixic acid $(30 \mu \mathrm{g})$ from Becton Dickinson, San Jose, California, USA. The zones of inhibition were measured in millimeters $(\mathrm{mm})$ and recorded.

\section{Minimum inhibitory concentration}

$S$. Typhi isolates that were resistant to nalidixic acid and ciprofloxacin by disc diffusion test had MIC performed for using an automated Vitek 2 compact system (bioMérieux, Inc., Durham NC, USA).

\section{Results}

A total of 70 isolates were MDR (resistant to ampicillin, cotrimoxazole and chloramphenicol) while 15 isolates were sensitive to all drugs tested.
Only one isolate was resistant to tetracycline alone while four isolates were multiply resistant to two drugs each, which included ampicillin, chloramphenicol, amoxycillin/clavulanic acid and tetracycline. Five isolates were resistant to five drugs, which included tetracycline, ampicillin, cotrimoxazole, chloramphenicol, and nalidixic acid. The most effective drugs were gentamicin, with 99 isolates being susceptible to the drug; this was followed by ceftriaxone in which 94 isolates were susceptible to the drug. Of the 100 isolates, 82 were sensitive to ciprofloxacin, 78 isolates were sensitive to cefuroxime, and 65 isolates were sensitive to nalidixic acid. The least effective drugs included tetracycline and ampicillin (only 24 isolates were sensitive to both drugs).

Resistance to individual antibiotics varied slightly during the study period. For instance, the prevalence of chloramphenical-resistant isolates, which was $81 \%$ in 2004 , reduced gradually to $67 \%$ by 2005 and slightly increased to $76 \%$ in 2006 . Similar trends were observed in ciprofloxacin, ampicillin, tetracycline, cotrimoxazole, nalidixic acid and ciprofloxacin. There was a gradual decrease in resistance in Augmentin observed over the study period.

The highest number of MDR $S$. Typhi was recorded in 2004, when $73 \%$ of the organisms isolated were MDR. There was no significant increase in MDR from 2005 (68\%) to 2006 (69\%) (Table 1).

Thirteen $S$. Typhi isolates that were resistant to ciprofloxacin and nalidixic acid by disk diffusion technique had MIC tests performed on them. The MICs of the 13 isolates ranged from 0.25 to 1.00 $\mu \mathrm{g} / \mathrm{ml}$. Eleven $S$. Typhi had an MIC of $0.25 \mu \mathrm{g} / \mathrm{ml}$ while two isolates had an MIC of $1.00 \mu \mathrm{g} / \mathrm{ml}$.

\section{Discussion}

Typhoid fever continues to pose a public health challenge in developing countries and the World Health Organization (WHO) estimates that the current annual global burden of typhoid is approximately 22 million new cases, $5 \%$ of which are fatal $[2,3]$. There has been an increasing concern about the prevalence of MDR $S$. Typhi strains in developing countries; several reports showing that MDR $S$. Typhi with plasmid-mediated resistance to conventional antibiotics including chloramphenicol, ampicillin, and contrimoxazole, abound in different parts of the developing world including Kenya [7]. This study examined a total of 70 MDR isolates of $S$. 
Table 1. Antimicrobial resistant Salmonella Typhi isolated from patients from 2004 - 2006 in Nairobi

\begin{tabular}{|l|l|l|l|l|l|l|l|l|l|l|l|l|}
\hline Year & $\begin{array}{l}\text { Number of } \\
\text { isolates }\end{array}$ & \multicolumn{9}{|c|}{ Number of isolates resistant to each antimicrobial (\%) } & No.of MDR \\
\hline & & Amp & Chlo & Tet & Cot & Nal & Cip & Ceft & Aug & gent & Cefu & \\
\hline 2004 & 32 & $88 \%$ & $81 \%$ & $75 \%$ & $75 \%$ & $31 \%$ & $19 \%$ & $13 \%$ & $81 \%$ & $0 \%$ & $16 \%$ & $68 \%$ \\
\hline 2005 & 39 & $64 \%$ & $67 \%$ & $69 \%$ & $67 \%$ & $38 \%$ & $18 \%$ & $0 \%$ & $62 \%$ & $3 \%$ & $3 \%$ & $68 \%$ \\
\hline 2006 & 29 & $76 \%$ & $76 \%$ & $38 \%$ & $72 \%$ & $34 \%$ & $14 \%$ & $0 \%$ & $62 \%$ & $0 \%$ & $0 \%$ & $69 \%$ \\
\hline Total & 100 & $75 \%$ & $74 \%$ & $62 \%$ & $73 \%$ & $35 \%$ & $18 \%$ & $40 \%$ & $68 \%$ & $1 \%$ & $6 \%$ & $70 \%$ \\
\hline
\end{tabular}

Typhi isolated from the Aga Khan University Hospital and the Kenyatta National Hospital. Previous studies have shown that the prevalence of MDR $S$. Typhi has been rising steadily from 50 to $65 \%$ in 1997 to 1999 [7] to the present level of $70 \%$ in this study. The rise in MDR could be attributed to an increase in misuse of drugs that are commonly available to the public for treatment of infections. Threlfall et al. (2001) established that in Britain, the increase in MDR to chloramphenicol, ampicillin, and trimethoprim increased from $21 \%$ in 1991 to $36 \%$ in 1994. The increasing levels of resistance were also reported by Kariuki et al. (2000) who established that from 1988 to 1993, all the $S$. Typhi isolated from Kenya were fully sensitive to all drugs used, which was in contrast to $82 \%$ of $S$. Typhi isolated in 1997 to 1999 which were resistant to ampicillin, tetracycline and chloramphenicol.

The emergence of MDR $S$. Typhi in Kenya is of concern because it limits the number of first-line antibiotics that are commonly used for treating typhoid as well other bacterial infections in hospitals. This poses a major challenge to health care. Currently, due to the increase of MDR strains in many parts of the world, the drugs of choice for treatment of $S$. Typhi are fluroquinolones. However, sporadic cases of ciprofloxacin treatment failure in typhoid fever have been reported in Europe and more recently, in Asia [10,11]. Resistance to nalidixic acid has also been reported in Cameroon where there has been a treatment failure to fluoroquinolones [12].

In this study, $S$. Typhi that were resistant to nalidixic acid were reported. Resistance in nalidixic acid was 31\% in 2004 and increased to $38 \%$ in 2005. Although ciprofloxacin is recommended as the drug of choice for treatment of MDR $S$. Typhi infections [13], a study by the National Institute of Health of Korea (NIHK) suggested that the clinical failure of empirical fluoroquinolone treatment might occur as the nalidixic acid resistance rate had rapidly increased from $3.5 \%$ in 2001 to $23.3 \%$ in 2002 in
Korea [14]. In the present study, $18 \%$ of the organisms were resistant to ciprofloxacin and $15 \%$ were recorded as intermediate by disk diffusion technique.

In conclusion, the high levels of resistance to commonly available antibiotics including fluroquinolones is of major public health concern in the treatment of typhoid fever and other bacterial infections in Kenya. To minimize the emergence and spread of resistance we recommend a prudent approach to the availability and usage of antibiotics in Kenya so that only qualified trained and medical personnel should administer antibiotics to patients. Also, the drug resistance in $S$. Typhi should be continuously monitored in Kenya to determine the extent of resistance to currently used drugs in the treatment of typhoid. There is also need to improve laboratory capacity detection and surveillance of typhoid. Despite improvements in health care facilities in Kenya, many areas still lack proper sanitation and good quality drinking water, which contributes to increased risk of transmission of all enteric pathogens. To minimize the emergence and spread of resistance we recommend a prudent approach to the availability and usage of antibiotics. Tools that are more specific and sensitive such as PCR should be adopted to improve surveillance for resistant strains of $S$. Typhi.

\section{Acknowledgements}

The authors gratefully acknowledge the assistance of Ms. Agnes Munyalo, Ms. Jane Muyodi, Mr. John Kiiru, Mr. Lawrence Thing'o, and Ms. Joyce Mwituria at the Centre for Microbiology Research, Kenya Medical Research Institute.

\section{References}

1. World Health Organization Department of Vaccines and Biologicals (2003) Background document: the diagnosis, prevention and treatment of typhoid fever. Geneva: 19-23.

2. Ivanoff, BCCL (2003) The diagnosis, prevention, and treatment of typhoid fever. World Health Organization, Geneva, Switzerland. 
3. Crump JA, Luby SP, Mintz ED (2004) The global burden of typhoid fever Bull. W.H.O. 82: 346-353.

4. Mirza, S H (2005) Multi-drug Resistant Typhoid: A Global Review. Infectious diseases Journal of Pakistan http://www.idspak.org/journal/2005/Jan-March/page1720.pdf.

5. Saha SK, Baqui AH, Hanif M, Darmstadt GL, Ruhulamin M, Nagatake T, Santosham M, Black R (2001) Typhoid fever in Bangladesh: implications for vaccination policy The Pediatric Infectious Disease Journal 20: 521-524.

6. Kariuki S, Gilks C, Revathi G, Hart CA (2000) Genotypic analysis of multidrug-resistant Salmonella enterica Serovar Typhi, Kenya. Emerging Infectious Diseases 6: 649-65.

7. Mirza SH, Kariuki S, Mamun KZ, Beeching NJ, Hart CA (2000) Analysis of plasmids and chromosomal DNA of multi-drug resistance Salmonella enterica serovar Typhi from Asia. Journal of Clinical Microbiology 44: 317-319

8. Kariuki S, Gilks CF, Kimani J, Muyodi J, Waiyaki P, Hart CA (2004) Plasmid diversity of multidrug resistance Escherichia coli isolated from children from a poultry farming area in Kenya. Annual Tropical Medical Parasitology 42: 1477 - 1482.

9. Parry CM (2004) Typhoid fever. Current Infectious Diseases Report 6, 27 - 33.

10. Parry CM, Hien TT, Daugan G, White NJ, Farrar J (2002) Typhoid fever. New England Journal of Medicine 347: 1770-1782.
11. Butt T, Ahmad RN, Mahmood A, Zaidi S (2003) Ciprofloxacin Treatment Failure in Typhoid Fever Case, Pakistan. Emerging Infectious Diseases 9: 1621-1622.

12. Njinkeng J, Nkemngu E, Asonganyi DN, Anna, LN (2005) Treatment failure in a typhoid patient infected with nalidixic acid resistant $S$. enterica serovar Typhi with reduced susceptibility to Ciprofloxacin: a case report from Cameroon. Biomedicine central infectious diseases 5 . http://www.biomedcentral.com/content/pdf/1471-2334-549.pdf.

13. Miller, SI, Pegues, DA (2000) "Salmonella species including Salmonella Typhi, in Mandell: andell GL, Bennett JE, Dolin R, editors. Principles and Practice of Infectious Diseases. 5th ed. Philadelphia: Churchill Livingstone, 234456.

14. Kyunwon L, Dongeun Y, Jong HY, Young S L, Hyun SK, Bok KL, Yunsop C (2004) Emergence of MultidrugResistant Salmonella enterica Serovar Typhi in Korea. Antimicrob. Agents. Chemother 11: 4130 - 4135.

\section{Corresponding author}

\section{M. Mengo}

Institute of Tropical Medicine and Infectious Diseases Jomo Kenyatta University of Agriculture and Technology PO Box 62000-00200

Nairobi, Kenya

Conflict of interests: No conflict of interests is declared. 